\title{
A NEW URN MODEL
}

\author{
MAY-RU CHEN, ${ }^{*}$ National Changhua University of Education \\ CHING-ZONG WEI,** Academia Sinica, Taiwan
}

\begin{abstract}
In this paper, we propose a new urn model. A single urn contains $b$ black balls and $w$ white balls. For each observation, we randomly draw $m$ balls and note their colors, say $k$ black balls and $m-k$ white balls. We return the drawn balls to the urn with an additional $c k$ black balls and $c(m-k)$ white balls. We repeat this procedure $n$ times and denote by $X_{n}$ the fraction of black balls after the $n$th draw. To investigate the asymptotic properties of $X_{n}$, we first perform some computational studies. We then show that $\left\{X_{n}\right\}$ forms a martingale, which converges almost surely to a random variable $X$. The distribution of $X$ is then shown to be absolutely continuous.
\end{abstract}

Keywords: Urn model; martingale; symmetric polynomial; absolute continuity for a random variable

2000 Mathematics Subject Classification: Primary 60E05

Secondary 60G42; 60F 15

\section{Introduction}

An urn model is constructed by imagining a number of urns, some or all containing balls of various colors. Sequences of experiments (trials) in which balls are drawn from and possibly returned to the urns according to certain rules are considered. These rules may include requirements for the addition to or removal of balls from certain urns at various stages of the experiment. Why have people investigated urn models? As explained by Kotz and Balakrishnan [10], there are three reasons. Firstly, urn models give an efficient way to describe the concept of 'random choice'. Secondly, urns and chance experiments can be compounded into new ones. Thirdly, as Pólya ably displayed over thirty years ago, an urn model allows a complex random (chance) process to be 'simulated'. Here, the term 'simulation' can be interpreted as a statistical equivalent to the mathematical concept of an isomorphism, which is essentially associated with urn models. As a result, an urn model plays a fundamental role in many problems.

The study of urn models has a long history. In 1657, Huygens proposed problems about urns in his treatise (see [11, pp. 48-55]), but he did not use the term 'urn'. James Bernoulli (1713) may have been the first person to mention problems in the language of urns (see [10]). Bernoulli discussed the problem, in the third book of his Ars Conjectandi, of drawing 'calculi' out of urns. The results on urn models up to 1977 were summarized in the book Urn Models and

Received 10 August 2004; revision received 14 March 2005.

* Postal address: Department of Mathematics, National Changhua University of Education, 1 Jin-De Road, Changhua, 500, Taiwan, Republic of China. Email address: mayru@ms29.url.com.tw

** Postal address: Institute of Statistical Science, Academia Sinica, 128 Academia Road, Sec. 2, Taipei, 115, Taiwan, Republic of China. Email address: czw@stat.sinica.edu.tw 
Their Application [9, pp. 176-181]. This book stimulated many probabilists and statisticians to investigate different kinds of urn model. After two decades, Kotz and Balakrishnan published the survey paper [10]. This covered almost all kinds of urn model and their properties.

This paper is organized as follows. In Section 2, a brief survey on Pólya-Eggenberger models, taken from published material, is given. The model formulations and the asymptotic properties of the corresponding urn models are especially emphasized. In Section 3, a new urn model that draws $m$ balls each time from an urn with black and white balls is proposed. Some computational studies are also conducted to compare the fitted beta distribution with the distribution of the fraction $X_{n}$ of black balls in our models. These are illustrated in figures. In Section $4,\left\{X_{n}\right\}$ is shown to form a martingale that converges almost surely (a.s.) to a random variable $X$. The absolute continuity of $X$ is then proved through combinatorial and algebraic arguments. The complete characterization of $X$ still remains to be resolved in the future. In the appendix, more figures are used to illustrate the effects of different initial numbers of black balls $b$ and white balls $w$, and different numbers of balls drawn $m$.

\section{The Pólya-Eggenberger urn model and its generalizations}

In this section, we will sketch the Pólya-Eggenberger urn model and its generalizations and modifications.

Eggenberger and Pólya [3], in 1923, proposed an urn model with a single urn initially containing $b$ black balls and $w$ white balls. A ball is drawn at random and then replaced together with $c$ balls of the same color. The procedure is repeated $n$ times and we denote by $X_{n}$ the proportion of black balls after the $n$th draw. It is known that $\left\{X_{n}\right\}$ is a martingale. Furthermore, $\lim _{n \rightarrow \infty} X_{n}=X$ exists, and $X$ has a beta distribution with parameters $b / c$ and $w / c$ (see [4, p. 226]).

In 1980, Hill et al. [8] proposed a generalized Pólya-Eggenberger urn model. It was the same as the Pólya-Eggenberger urn model except that the probability of drawing balls, instead of being $B_{n} /\left(W_{n}+B_{n}\right)$, was $f\left(B_{n} /\left(W_{n}+B_{n}\right)\right)$, where $B_{n}$ and $W_{n}$ denote the number of black balls and white balls, respectively, at the $n$th stage, and $f$ is any function mapping $[0,1]$ into itself. They showed that, under a condition on $f$ at $p_{0}$, where $p_{0} \in\{x: f(x)=x\}$, the fraction of black balls converges to $p_{0}$ with positive probability. Moreover, if $p_{0}$ is an unstable fixed point of $f$ (i.e. $f\left(p_{0}\right)=p_{0}$ and, in some neighborhood of $p_{0}, f(x)<x$ for $x<p_{0}$ and $f(x)>x$ for $\left.x>p_{0}\right)$ then $\mathrm{P}\left(B_{n} /\left(W_{n}+B_{n}\right) \rightarrow p_{0}\right)=0$.

In 1985, Bagchi and Pal [1] defined a tenable Pólya-Eggenberger urn model which described a single urn with $b_{0}$ black balls and $t_{0}-b_{0}$ white balls, where $b_{0}>1$ and $t_{0}>1$. A ball is drawn at random. Its color is noted and the ball is returned to the urn. If the color is black, then $a$ black balls and $b>0$ white balls are added to the urn. Otherwise, $c>0$ black balls and $d$ white balls are added to the urn. Now, let $a+b=c+d \geq 1, a \neq c$. For the tenable urn process, Gouet [5] used martingale arguments to show that if $\max (b, c)>0$, then the fraction of black balls converges almost surely to $c /(b+c)$. Four years later, by using martingale transforms and the functional central limit theorem for the tenable urn process, Gouet [6] provided weak convergence results for the sequence of processes $B_{k_{n}(t)}-c\left(B_{k_{n}(t)}+W_{k_{n}(t)}\right) /(b+c), n \in \mathbb{N}$, $t \geq 0$, where $\left\{k_{n}(t)\right\}$ is a sequence of deterministic time scales.

In 1990, Pemantle [12] generalized the Pólya-Eggenberger urn model by replacing $c$ with a function of time. He showed that the proportions of black balls converges a.s. and the limit has no atoms except possibly at 0 or 1 . Necessary and sufficient conditions for the limit to concentrate entirely on the set $\{0,1\}$ were given. 
In 2001, Schreiber [13] studied the asymptotic behavior of a class of generalized PólyaEggenberger urn model by using an ordinary differential equation (ODE) method. He also formulated a generalized Pólya-Eggenberger urn model called the replicator process.

Two balls are selected at random with replacement from an urn and new balls are added or removed, at varying rates, according to probabilities that depend only on the colors of the chosen balls.

Schreiber proved that

(i) the number of balls increases asymptotically at a linear rate,

(ii) the distribution $x(n)$ of the strategies at the $n$th update is a noisy Cauchy-Euler approximation to the mean limit ODE of the process, and

(iii) the limit set of $x(n)$ is a.s. a connected, internally chain-recurrent set for the mean limit ODE.

\section{A new urn model and some computational studies}

In this section, we briefly introduce the Pólya-Eggenberger urn model and its generalizations and modifications. Drawing one ball at random each time is the common property of these models. We now introduce a new model in which more than one ball is drawn randomly each time.

Model M. A single urn contains $b$ black balls and $w$ white balls. We draw $m$ balls at random, say $k$ black balls and $m-k$ white balls, and their colors are noted. Return these balls to the urn with $c k$ black balls and $c(m-k)$ white balls. Repeat the procedure $n$ times.

To gain some insight into this new model, we perform some computational studies. Let $Y_{n}$ be the number of black balls after the $n$th draw. Using the Markov property of $\left\{Y_{n}\right\}$ (see (4.1), below), the probability $\mathrm{P}\left(Y_{n}=k\right)$ is computed recursively. The result is shown as solid curves in Figures 1 and 2. The dashed curves show the density of the fitted beta distribution. Here, the parameters of the beta distributions are estimated by the least-squares method assuming that $\left\{\mathrm{P}\left(Y_{n}=k\right)(b+w+c m n)\right\}$ is the data set. All programs are executed using MAPLE ${ }^{\circledR} 6$.

The graphs for the fractions of black balls are obtained under the condition $c=1$. In Figures 1 and 2, we denote our new model by $\mathbf{M}(b, w, m)$ and a beta distribution with parameters $\alpha$ and $\beta$ by beta $(\alpha, \beta)$. Figures 1 and 2 demonstrate the effects of different values of $b, w$, and $m$. More experiments are given in the appendix. Note that in Figures 1 and 2 the values of $b$ are less than or equal to $w$. If we were to exchange the values of $b$ and $w$, the new graphs would be symmetric with the old ones with respect to the line $x=0.5$. Therefore, we do not consider the cases with $b>w$.

In Figure 1, we can see that the peak of the curve is higher and skews to the left if the value of $w$ increases and $w \geq b$. This is reasonable since the probability of drawing white balls is getting larger and the fraction of black balls is getting smaller.

If we compare Figure 1(a) with Figure 2, it is easy to see that the peaks get higher and higher as $b$ increases. Moreover, if $b=w=m$ then $\mathrm{M}(b, b, b)$ is close to $\operatorname{beta}(\alpha, \alpha)$ for some $\alpha$. It 
(a)

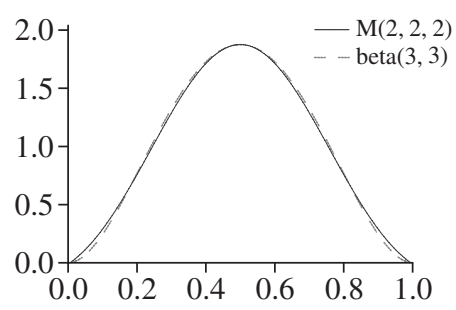

(b)

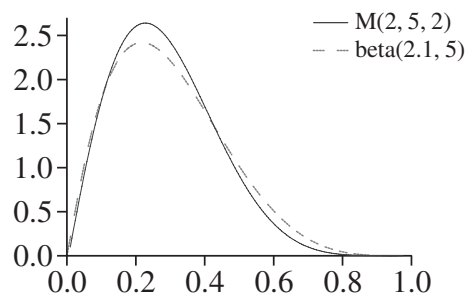

FIGURE 1: Two examples with $n=100$.

(a)

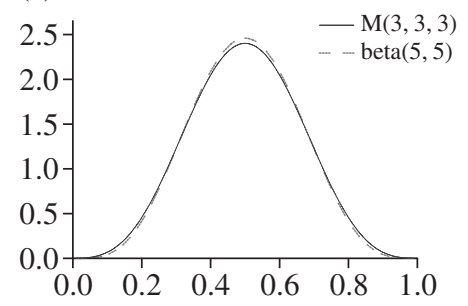

(b)

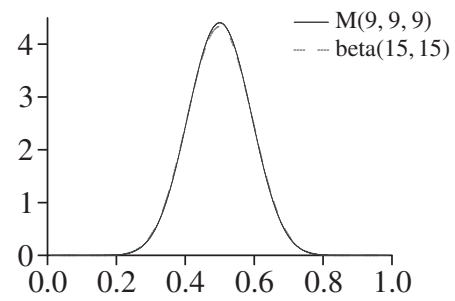

FIGURE 2: Two examples with $n=100$.

would be of interest to see the relationship between $b$ and $\alpha$. Figures 1 and 2 seem to indicate that the general limiting distributions for $m>1$ may not be beta distributed, as they are for $m=1$. Although they cannot help us in guessing the exact behavior of the limiting distribution at this moment, they do provide some insight. For example, they indicate the existence of the limiting distributions and the densities. These will be shown in Section 4.

\section{Martingale property and absolute continuity of the limit}

In this section, we show that in Model $M$ the fraction of black balls forms a martingale. This martingale converges a.s. to a limit $X$. We then prove that $X$ is absolutely continuous.

\subsection{Martingale}

Recall our new urn model defined in Section 3. Let $Y_{n}$ be the number of black balls after $n$ draws and let $X_{n}=Y_{n} / \gamma_{n}$ be the fraction of black balls, where $\gamma_{n}=b+w+c m n$ is the total number of balls after $n$ draws. Also, let $\mathcal{F}_{n}$ be the $\sigma$-field generated by $Y_{1}, Y_{2}, \ldots, Y_{n}$.

Theorem 4.1. For Model $M,\left\{X_{n}, \mathcal{F}_{n}, n \geq 1\right\}$ is a martingale, i.e. $\mathrm{E}\left[X_{n+1} \mid \mathcal{F}_{n}\right]=X_{n}$. Furthermore, $X_{n}$ converges a.s. to a random variable $X$ with $0 \leq X \leq 1$.

Proof. It is clear that

$$
\mathrm{P}\left(Y_{n+1}=j \mid Y_{n}=k, Y_{n-1}=k_{n-1}, \ldots, Y_{0}=b\right)=\mathrm{P}\left(Y_{n+1}=j \mid Y_{n}=k\right) .
$$

Thus, $\left\{Y_{n}\right\}$ is a discrete-time Markov process, and so is $\left\{X_{n}\right\}$. Since $\left\{X_{n}\right\}$ is a discrete-time Markov process, $\mathrm{E}\left[X_{n+1} \mid \mathcal{F}_{n}\right]=\mathrm{E}\left[X_{n+1} \mid Y_{n}\right]$. Since $0 \leq X_{n} \leq 1,\left\{X_{n}, \mathcal{F}_{n}, n \geq 1\right\}$ is a martingale if we can prove that

$$
\mathrm{E}\left[X_{n+1} \mathbf{1}_{\left\{Y_{n}=k\right\}}\right]=\mathrm{E}\left[X_{n} \mathbf{1}_{\left\{Y_{n}=k\right\}}\right],
$$


for $k=b, b+1, \ldots, b+c m n$, where $\mathbf{1}_{\{\cdot\}}$ is the indicator function. By definition,

$$
\begin{aligned}
\mathrm{E}\left[X_{n+1} \mathbf{1}_{\left\{Y_{n}=k\right\}}\right] & =\sum_{\ell=0}^{m} \frac{k+\ell c}{\gamma_{n+1}} \mathrm{P}\left(Y_{n+1}=k+\ell c \mid Y_{n}=k\right) \mathrm{P}\left(Y_{n}=k\right) \\
& =\sum_{\ell=0}^{m} \frac{k+\ell c}{\gamma_{n+1}}\left(\begin{array}{c}
k \\
\ell
\end{array}\right)\left(\begin{array}{c}
\gamma_{n}-k \\
m-\ell
\end{array}\right)\left(\begin{array}{c}
\gamma_{n} \\
m
\end{array}\right)^{-1} \mathrm{P}\left(Y_{n}=k\right),
\end{aligned}
$$

where

$$
\left(\begin{array}{l}
n \\
m
\end{array}\right)= \begin{cases}\frac{n !}{m !(n-m) !} & \text { if } n \geq m \\
0 & \text { if } n<m\end{cases}
$$

To simplify (4.2), we need the following two identities:

$$
\begin{aligned}
& \sum_{\ell=0}^{m}\left(\begin{array}{l}
k \\
\ell
\end{array}\right)\left(\begin{array}{l}
\gamma_{n}-k \\
m-\ell
\end{array}\right)=\left(\begin{array}{l}
\gamma_{n} \\
m
\end{array}\right), \\
& \sum_{\ell=0}^{m} \ell\left(\begin{array}{l}
k \\
\ell
\end{array}\right)\left(\begin{array}{l}
\gamma_{n}-k \\
m-\ell
\end{array}\right)=k\left(\begin{array}{l}
\gamma_{n}-1 \\
m-1
\end{array}\right) .
\end{aligned}
$$

Here, (4.3) can be derived by comparing the coefficients of the term $x^{m}$ in the expressions of both sides of the identity $(1+x)^{k}(1+x)^{\gamma_{n}-k}=(1+x)^{\gamma_{n}}$. Identity (4.4) can then be derived from (4.3). If we simplify (4.2) using (4.3) and (4.4), then we obtain

$$
\begin{aligned}
\mathrm{E}\left[X_{n+1} \mathbf{1}_{\left\{Y_{n}=k\right\}}\right] & =\mathrm{P}\left(Y_{n}=k\right)\left[k\left(\begin{array}{l}
\gamma_{n} \\
m
\end{array}\right)+k c\left(\begin{array}{l}
\gamma_{n}-1 \\
m-1
\end{array}\right)\right]\left[\gamma_{n+1}\left(\begin{array}{l}
\gamma_{n} \\
m
\end{array}\right)\right]^{-1} \\
& =\frac{\mathrm{P}\left(Y_{n}=k\right) k}{\gamma_{n}} \\
& =\mathrm{E}\left[X_{n} \mathbf{1}_{\left\{Y_{n}=k\right\}}\right] .
\end{aligned}
$$

The last identity holds since $X_{n}=k / \gamma_{n}$ when $Y_{n}=k$. Since $0 \leq X_{n} \leq 1, n=1,2, \ldots$, we can apply the martingale convergence theorem (see [7, p. 18]) to claim that there exists an $X, 0 \leq X \leq 1$, such that $X_{n} \stackrel{\text { a.s. }}{\longrightarrow} X$ as $n \rightarrow \infty$.

Remark 4.1. When $m=1$, our model becomes the classical Pólya-Eggenberger urn model. It has been proved that $X_{n} \stackrel{\text { a.s. }}{\longrightarrow} X$ and $X$ has a beta distribution with parameters $b / c$ and $w / c$ (see [9]). Theorem 4.1 can be viewed as a generalization of this result.

Remark 4.2. Let Model M be modified as follows. 'A single urn contains balls of $r$ different colors. For each color $i$, we initially have $b_{i}$ balls. We draw $m$ balls at random, say $k_{i}$ balls of color $i$, and their colors are noted. Return these balls to the urn with $c k_{i}$ balls of color $i$. Repeat the procedure $n$ times.' Then Theorem 4.1 can be viewed as results about the fraction of balls of the first color for an urn model with two colors. Similarly, if we replace $Y_{n}$ by $Y_{n, i}$ and $X_{n}$ by $X_{n, i}$ in the proof of Theorem 4.1, we find that, for all $i=1, \ldots, r,\left\{X_{n, i}, \mathcal{F}_{n}, n \geq 1\right\}$ is a martingale and converges a.s. Therefore, $X_{n}=\left(X_{n, 1}, \ldots, X_{n, r}\right)$ is also a martingale and converges a.s. to a random variable $X=\left(X_{1}, \ldots, X_{r}\right)$, with $0 \leq X_{i} \leq 1$ a.s. for $i=1, \ldots, r$. 


\subsection{Absolute continuity}

To understand the asymptotic performance of Model M, the distribution of $X$ is definitely of interest. Unfortunately, we are not able to characterize the distribution of $X$ at this moment. Instead, we will prove that $X$ is absolutely continuous. That is, we will prove that the distribution of $X$ has a density. This is the content of the following theorem.

Theorem 4.2. For fixed $b, w, c$, and $m, X$ is absolutely continuous.

The analytical complexity of proving the absolute continuity is somewhat greater than that of proving the continuity of the distribution (see [15]). We adopt the following approach. Let $(\Omega, \mathcal{F}, \mathrm{P})$ be the probability space. We first find a sequence of events $\Omega_{\ell}$ such that $\Omega_{\ell} \subset \Omega_{\ell+1}$ and $\mathrm{P}\left(\bigcup_{\ell=1}^{\infty} \Omega_{\ell}\right)=1$. Then, by restricting $X$ to $\Omega_{\ell}$, we show that it has a density $f_{\ell}$. Finally, we prove that $f=\lim _{\ell \rightarrow \infty} f_{\ell}$ exists and that $f$ is the density of $X$.

We now show that our approach is valid using the following propositions.

Proposition 4.1. Let $\Omega_{\ell}$ be a sequence of increasing events such that $\mathrm{P}\left(\bigcup_{\ell=1}^{\infty} \Omega_{\ell}\right)=1$. If there exist nonnegative Borel measurable functions $\left\{f_{\ell}\right\}_{\ell \geq 1}$ such that $\mathrm{P}\left(\Omega_{\ell} \cap X^{-1}(B)\right)=\int_{B} f_{\ell}(x) \mathrm{d} x$ for all Borel sets $B$, then $f=\lim _{\ell \rightarrow \infty} f_{\ell}$ exists almost everywhere (a.e.) and $f$ is the density of $X$.

Proof. Since $\Omega_{\ell+1} \supset \Omega_{\ell}$, for all Borel sets $B$ we obtain

$$
\int_{B} f_{\ell+1}(x) \mathrm{d} x=\mathrm{P}\left(\Omega_{\ell+1} \cap X^{-1}(B)\right) \geq \mathrm{P}\left(\Omega_{\ell} \cap X^{-1}(B)\right)=\int_{B} f_{\ell}(x) \mathrm{d} x .
$$

This implies that $f_{\ell+1} \geq f_{\ell} \geq 0$ a.e. Let $f=\lim _{\ell \rightarrow \infty} f_{\ell}$, where $0 \leq f \leq \infty$. The monotone convergence theorem (see [16]) ensures that $\int_{B} f(x) \mathrm{d} x=\lim _{\ell \rightarrow \infty} \int_{B} \overline{f_{\ell}}(x) \mathrm{d} x$ for all Borel sets $B$. Moreover, since $\mathrm{P}\left(\bigcup_{\ell=1}^{\infty} \Omega_{\ell}\right)=1$,

$$
\int_{\mathbb{R}} f(x) \mathrm{d} x=\lim _{\ell \rightarrow \infty} \int_{\mathbb{R}} f_{\ell}(x) \mathrm{d} x=\lim _{\ell \rightarrow \infty} \mathrm{P}\left(\Omega_{\ell}\right)=1 .
$$

Therefore, $f<\infty$ a.e. and $X$ is absolutely continuous with density $f$.

The following proposition provides a way to construct the events $\Omega_{\ell}$.

Proposition 4.2. For fixed $b, w, c$, and $m$, let

$$
\Omega_{\ell}=\left\{\omega: Y_{\ell}(\omega) \geq c m \text { and } \gamma_{\ell}-Y_{\ell}(\omega) \geq c m\right\},
$$

where $\gamma_{n}=b+w+$ cmn and $\ell \geq 1$. Then $\Omega_{\ell+1} \supset \Omega_{\ell}$ and $\mathrm{P}\left(\bigcup_{\ell=1}^{\infty} \Omega_{\ell}\right)=1$.

Proof. The assertion $\Omega_{\ell+1} \supset \Omega_{\ell}$ is obvious from the fact that the number of black balls $Y_{\ell}$ and the number of white balls $\gamma_{\ell}-Y_{\ell}$ are increasing with $\ell$. For the second assertion, it is equivalent to show that $\mathrm{P}\left(Y_{\ell} \geq \mathrm{cm}\right.$ and $\gamma_{\ell}-Y_{\ell} \geq \mathrm{cm}$ for some $\left.\ell\right)=1$. Observe that it is sufficient to prove that $\mathrm{P}\left(Y_{\ell}<\mathrm{cm}\right.$ for all $\left.\ell\right)=0$ and $\mathrm{P}\left(\gamma_{\ell}-Y_{\ell}<\mathrm{cm}\right.$ for all $\left.\ell\right)=0$. In fact, we only have to prove that

$$
\mathrm{P}\left(Y_{\ell}<\mathrm{cm} \text { for all } \ell\right)=0 .
$$

This is because, by exchanging the roles of black balls and white balls, the second statement can be proved similarly. 
Consider the events $E_{n}$, 'at least one black ball is drawn in the sample of size $m$ at time $n$ ', and $F_{n}$, 'the first ball drawn in the sample of size $m$ at time $n$ is black'. Then

$$
\mathrm{P}\left(Y_{\ell}<c m \text { for all } \ell\right) \leq 1-\mathrm{P}\left(E_{n} \text { infinitely often }\right)
$$

From the conditional Borel-Cantelli lemma (see [7, p. 32]), we have $\mathrm{P}\left(E_{n}\right.$ infinitely often $)=1$ if $\sum_{n} \mathrm{P}\left(E_{n+1} \mid \mathcal{F}_{n}\right)=\infty$ a.s. Since

$$
\mathrm{P}\left(E_{n+1} \mid \mathcal{F}_{n}\right) \geq \mathrm{P}\left(F_{n+1} \mid \mathcal{F}_{n}\right)=\frac{Y_{n}}{b+w+c m n} \geq \frac{b}{b+w+c m n} \quad \text { a.s. }
$$

and $\sum_{n}(b /(b+w+c m n))=\infty$, we have $\sum_{n} \mathrm{P}\left(E_{n+1} \mid \mathcal{F}_{n}\right)=\infty$ a.s. Consequently, the claim is proved.

Next, we will show that by restricting $X$ to $\Omega_{\ell}, X$ has a density. It is clear that this will hold if we can show that the restriction of $X$ to $\Omega_{\ell j}=\left\{\omega: Y_{\ell}(\omega)=j\right\}$ has a density for each $j$, with $\mathrm{cm} \leq j \leq \gamma_{\ell-1}$. For this, we first need the following lemmas.

Lemma 4.1. For $c m \leq j \leq \gamma_{\ell-1}, n \geq \ell$, and $k \leq m(n+1)$,

$$
\sum_{i=0}^{m}\left(\begin{array}{c}
j+c(k-i) \\
i
\end{array}\right)\left(\begin{array}{c}
\gamma_{n}-j-c(k-i) \\
m-i
\end{array}\right)=\frac{\gamma_{n}^{m}}{m !}+\frac{(1-m-2 c) \gamma_{n}^{m-1}}{2(m-1) !}+\cdots
$$

which is a polynomial in $\gamma_{n}$ of degree $m$ with coefficients depending on $b, w, m$, and conly.

Proof. Since the proof is a little complex, we use two steps.

Step 1. For $x, y \in \mathbb{R}$, define

$$
C_{i}^{x}= \begin{cases}1 & \text { if } i=0 \\ x(x-1) \cdots \frac{(x-i+1)}{i !} & \text { if } i>0\end{cases}
$$

and

$$
f_{m}(x, y)=\sum_{i=0}^{m} C_{i}^{x-c i} C_{m-i}^{y-c(m-i)}
$$

Note that $f_{m}(x, y)$ is a polynomial symmetric in $x$ and $y$. We claim that, for $m, c \in \mathbb{N}$,

$$
f_{m}(x, y) \in \mathbb{Z}[x+y],
$$

where $\mathbb{Z}[x+y]$ is the ring of polynomials over $\mathbb{Z}$ in the indeterminate $x+y$ (see [14] for a definition). The remaining part of this step is to prove (4.6).

Now we proceed to prove (4.6) by induction. For $m=1$, the claim is true since $\sum_{i=0}^{1} C_{i}^{x-c i} C_{1-i}^{y-c(1-i)}=x+y-2 c$. Suppose that (4.6) holds for $m=n-1$, where $n \in \mathbb{N}$. We use the identity

$$
C_{i}^{z}=C_{i-1}^{z-1}+C_{i}^{z-1}=C_{i-1}^{z-1}+C_{i-1}^{z-2}+C_{i}^{z-2}=C_{i-1}^{z-1}+C_{i-1}^{z-2}+\cdots+C_{i-1}^{z-c}+C_{i}^{z-c},
$$


which holds for $z \in \mathbb{R}$, to obtain

$$
\begin{aligned}
f_{n}(x, y) & =\sum_{i=0}^{n} C_{i}^{x-c i} C_{n-i}^{y-c(n-i)} \\
& =\sum_{i=1}^{n} \sum_{j=1}^{c} C_{i-1}^{x-c i-j} C_{n-i}^{y-c(n-i)}+\sum_{i=0}^{n} C_{i}^{(x-c)-c i} C_{n-i}^{y-c(n-i)} \\
& =\sum_{j=1}^{c} f_{n-1}(x-c-j, y)+f_{n}(x-c, y) .
\end{aligned}
$$

Replacing $y$ by $y+c$ in (4.7), we obtain

$$
f_{n}(x, y+c)=\sum_{j=1}^{c} f_{n-1}(x-c-j, y+c)+f_{n}(x-c, y+c) .
$$

Then

$$
f_{n}(x-c, y+c)=f_{n}(x, y+c)-\sum_{j=1}^{c} f_{n-1}(x-c-j, y+c) .
$$

Since $f_{n}(x, y)=f_{n}(y, x)$, we obtain

$$
f_{n}(x-c, y+c)=f_{n}(y+c, x)-\sum_{j=1}^{c} f_{n-1}(x-c-j, y+c) .
$$

By replacing $x$ by $y+c$ and $y$ by $x$ in (4.7), we have

$$
f_{n}(y+c, x)=\sum_{j=1}^{c} f_{n-1}(y-j, x)+f_{n}(y, x) .
$$

Therefore, by (4.8) and (4.9),

$$
f_{n}(x-c, y+c)=f_{n}(y, x)+\sum_{j=1}^{c}\left(f_{n-1}(y-j, x)-f_{n-1}(x-c-j, y+c)\right) .
$$

Now, by the induction hypothesis, (4.6) holds for $m=n-1$, i.e. there exists a polynomial $g_{n-1}$ (in a single variable) such that $g_{n-1}(x+y)=f_{n-1}(x, y)$. Hence, for $1 \leq j \leq c$,

$$
f_{n-1}(y-j, x)=g_{n-1}(x+y-j)=f_{n-1}(x-c-j, y+c) .
$$

In view of (4.10) and (4.11), we have

$$
f_{n}(x-c, y+c)=f_{n}(y, x)=f_{n}(x, y) .
$$

Let $h_{n}(x, y)=f_{n}(x, y)-f_{n}(\mathrm{~cm}, x+y-c m)$. To finish the induction proof, we are going to show that, as a polynomial in $x$ and $y, h_{n}(x, y)=0$.

Note that, for a fixed $x, h_{n}(x, y)$ is a polynomial in $y$ with degree at most $n$. Hence, we can write $h_{n}(x, y)=a_{n}^{n}(x) y^{n}+a_{n}^{n-1}(x) y^{n-1}+\cdots+a_{n}^{0}(x)$, where $a_{n}^{i}(x)$ is a polynomial 
in $x$ with a degree of at most $n, 0 \leq i \leq n$. Let $x_{0}$ be a multiple of $c$. Then, by (4.12), $f_{n}\left(x_{0}, y\right)=f_{n}\left(\mathrm{~cm}, x_{0}+y-\mathrm{cm}\right)$ and, consequently, $h_{n}\left(x_{0}, y\right)=0$. This in turn implies that $a_{n}^{n}\left(x_{0}\right)=0, a_{n}^{n-1}\left(x_{0}\right)=0, \ldots, a_{n}^{0}\left(x_{0}\right)=0$ for all $x_{0}$. Since there are infinitely many such $x_{0} \mathrm{~s}$,

$$
a_{n}^{n}(x)=0, a_{n}^{n-1}(x)=0, \ldots, a_{n}^{0}(x)=0,
$$

for all $x \in \mathbb{R}$. This implies that $h_{n}(x, y)$ is a zero polynomial.

Step 2. We substitute $x$ by $j+c k$ and $y$ by $\gamma_{n}-j-c k+c m$ in (4.5). Then

$\sum_{i=0}^{m} C_{i}^{j+c(k-i)} C_{m-i}^{\gamma_{n}-j-c(k-i)}=a_{m} \gamma_{n+1}^{m}+a_{m-1} \gamma_{n+1}^{m-1}+\cdots+a_{0}:=b_{m} \gamma_{n}^{m}+b_{m-1} \gamma_{n}^{m-1}+\cdots+b_{0}$.

In order to obtain the coefficients $b_{m}$ and $b_{m-1}$, let $k=0$ in $\sum_{i=0}^{m} C_{i}^{j+c(k-i)} C_{m-i}^{\gamma_{n}-j-c(k-i)}$. Then

$$
\begin{aligned}
\sum_{i=0}^{m} C_{i}^{j+c(k-i)} C_{m-i}^{\gamma_{n}-j-c(k-i)} & =C_{m}^{\gamma_{n}-j}+C_{1}^{j-c} C_{m-1}^{\gamma_{n}-j+c}+\cdots \\
& =\frac{\gamma_{n}^{m}}{m !}+\frac{(1-m-2 c) \gamma_{n}^{m-1}}{2(m-1) !}+\cdots
\end{aligned}
$$

Note that $j+c(k-i) \geq 0$ and $\gamma_{n}-j-c(k-i) \geq 0$, since $c m \leq j \leq \gamma_{\ell-1}, n \geq \ell$, and $k \leq m(n+1)$. Therefore,

$$
\sum_{i=0}^{m} C_{i}^{j+c(k-i)} C_{m-i}^{\gamma_{n}-j-c(k-i)}=\sum_{i=0}^{m}\left(\begin{array}{c}
j+c(k-i) \\
i
\end{array}\right)\left(\begin{array}{c}
\gamma_{n}-j-c(k-i) \\
m-i
\end{array}\right) .
$$

Hence, Lemma 4.1 holds.

Lemma 4.2. For a fixed $\ell$, there exists a positive constant $c^{\prime}$ such that, for all $n \geq \ell, j$ with $\mathrm{cm} \leq j \leq \gamma_{\ell-1}$, and $k \leq m(n+1)$, the following inequality holds:

$$
\sum_{i=0}^{m} \mathrm{P}\left(Y_{n+1}=j+c k \mid Y_{n}=j+c(k-i)\right) \leq 1-\frac{1}{n}+\frac{c^{\prime}}{n^{2}} .
$$

Proof. By Lemma 4.1, we have

$$
\begin{aligned}
\left(\sum_{i=0}^{m}\right. & \left(\begin{array}{c}
j+c(k-i) \\
i
\end{array}\right)\left(\begin{array}{c}
\gamma_{n}-j-c(k-i) \\
m-i
\end{array}\right)\left(\begin{array}{l}
\gamma_{n} \\
m
\end{array}\right)^{-1} \\
& =\left(\frac{\gamma_{n}^{m}}{m !}+\frac{(1-m-2 c) \gamma_{n}^{m-1}}{2(m-1) !}+\cdots\right)\left(\frac{\gamma_{n}^{m}}{m !}+\frac{(1-m) \gamma_{n}^{m-1}}{2(m-1) !}+\cdots\right)^{-1} \\
& =1-\frac{1}{n}+O\left(\frac{1}{n^{2}}\right)
\end{aligned}
$$

Hence, there exists a constant $c^{\prime}$ such that (4.13) holds for all $n$.

Now we are ready to prove Theorem 4.2. 
Proof of Theorem 4.2. Let $F$ be the distribution function of $X$. Our goal is to prove that $F$ is absolutely continuous. By Propositions 4.1 and 4.2 and the discussion before Lemma 4.1, it is sufficient to show that the restriction of $X$ to $\Omega_{\ell j}=\left\{\omega: Y_{\ell}(\omega)=j\right\}$ has a density for $c m \leq j \leq \gamma_{\ell-1}$.

Now, in view of Lemma 4.2, there exists a positive constant $c^{\prime}$ such that, for a fixed $\ell$ and for all $n \geq 0$,

$$
\begin{aligned}
\max _{0 \leq k \leq m(n+1)} \mathrm{P}\left(Y_{\ell+n+1}=j+c k \mid Y_{\ell}=j\right) & \\
\leq & \max _{0 \leq k \leq m(n+1)}\left\{\sum_{i=0}^{m} \mathrm{P}\left(Y_{\ell+n+1}=j+c k \mid Y_{\ell+n}=j+c(k-i)\right)\right. \\
& \left.\times \mathrm{P}\left(Y_{\ell+n}=j+c(k-i) \mid Y_{\ell}=j\right)\right\} \\
\leq & \max _{0 \leq k \leq m(n+1)}\left\{\sum_{i=0}^{m} \mathrm{P}\left(Y_{\ell+n+1}=j+c k \mid Y_{\ell+n}=j+c(k-i)\right)\right. \\
= & \max _{0 \leq k \leq m(n+1)} \sum_{i=0}^{m} \mathrm{P}\left(Y_{\ell+n+1}=j+c k \mid Y_{\ell+n}=j+c(k-i)\right) \\
& \times \max _{0 \leq i \leq m n} \mathrm{P}\left(Y_{\ell+n}=j+c i \mid Y_{\ell}=j\right) \\
\leq & {\left[1-\frac{1}{\ell+n}+\frac{c^{\prime}}{(\ell+n)^{2}}\right] \max _{0 \leq k \leq m n} \mathrm{P}\left(Y_{\ell+n}=j+c k \mid Y_{\ell}=j\right) . }
\end{aligned}
$$

This implies that, for a fixed $\ell$ and for all $n \geq \ell+1$,

$$
\begin{aligned}
& \max _{0 \leq k \leq m(n-\ell)} \mathrm{P}\left(Y_{n}=j+c k \mid Y_{\ell}=j\right) \\
& \leq {\left[1-\frac{1}{n-1}+\frac{c^{\prime}}{(n-1)^{2}}\right] \max _{0 \leq k \leq m(n-1-\ell)} \mathrm{P}\left(Y_{n-1}=j+c k \mid Y_{\ell}=j\right) } \\
& \leq {\left[1-\frac{1}{n-1}+\frac{c^{\prime}}{(n-1)^{2}}\right]\left[1-\frac{1}{n-2}+\frac{c^{\prime}}{(n-2)^{2}}\right] } \\
& \quad \times \max _{0 \leq k \leq m(n-2-\ell)} \mathrm{P}\left(Y_{n-2}=j+c k \mid Y_{\ell}=j\right) \\
& \vdots \quad \vdots \\
& \leq \prod_{i=\ell}^{n-1}\left(1-\frac{1}{i}+\frac{c^{\prime}}{i^{2}}\right) \\
& \leq c^{\prime \prime} \exp \left(-\sum_{i=\ell}^{n-1} \frac{1}{i}\right)
\end{aligned}
$$

for some positive constant $c^{\prime \prime}$. The last inequality can be derived from the fact that $1-x \leq \mathrm{e}^{-x}$. 
Therefore, for any $x$ and $x^{\prime}, 0 \leq x<x^{\prime} \leq 1$,

$$
\begin{aligned}
\mathrm{P}\left(x \leq X_{n} \leq x^{\prime} \mid Y_{\ell}=j\right) & =\mathrm{P}\left(x \gamma_{n} \leq Y_{n} \leq x^{\prime} \gamma_{n} \mid Y_{\ell}=j\right) \\
& \leq\left[\left(x^{\prime}-x\right) \gamma_{n}+1\right] c^{\prime \prime} \exp \left(-\sum_{i=\ell}^{n-1} \frac{1}{i}\right) .
\end{aligned}
$$

We obtain

$$
\begin{aligned}
\limsup _{n} \mathrm{P}\left(x \leq X_{n} \leq x^{\prime} \mid Y_{\ell}=j\right) & \leq \lim _{n \rightarrow \infty}\left[\left(x^{\prime}-x\right) \gamma_{n}+1\right] c^{\prime \prime} \exp \left(-\sum_{i=\ell}^{n-1} \frac{1}{i}\right) \\
& =\left(x^{\prime}-x\right) c^{\prime \prime \prime},
\end{aligned}
$$

where $c^{\prime \prime \prime}$ is a positive constant depending only on $\ell$. Now fix $\ell$. Let $\mathrm{cm} \leq j \leq \gamma_{\ell-1}$. For any given $\varepsilon>0$, let $\delta=\varepsilon / c^{\prime \prime \prime}$. Let $x_{1}<x_{1}^{\prime} \leq x_{2}<x_{2}^{\prime} \leq \cdots \leq x_{s}<x_{s}^{\prime}$ and $\sum_{i=1}^{s}\left|x_{i}^{\prime}-x_{i}\right|<\delta$. We have, by Fatou's lemma (see [16]),

$$
\begin{aligned}
\sum_{i=1}^{s} \mathrm{P}\left(\left\{x_{i}<X<x_{i}^{\prime}\right\} \cap \Omega_{\ell j}\right) & \leq \sum_{i=1}^{s} \liminf _{n \rightarrow \infty} \mathrm{E}\left[\mathbf{1}_{\left\{x_{i} \leq X_{n} \leq x_{i}^{\prime}\right\}} \mid Y_{\ell}=j\right] \mathrm{P}\left(\Omega_{\ell j}\right) \\
& \leq \sum_{i=1}^{s} \liminf _{n \rightarrow \infty} \mathrm{P}\left(x_{i} \leq X_{n} \leq x_{i}^{\prime} \mid Y_{\ell}=j\right) \mathrm{P}\left(\Omega_{\ell j}\right) \\
& \leq \sum_{i=1}^{s}\left(x_{i}^{\prime}-x_{i}\right) c^{\prime \prime \prime} \\
& <\varepsilon .
\end{aligned}
$$

By [2, Theorem 31.7], the restriction of $X$ to $\Omega_{\ell j}$ thus has a density. Hence, the proof is complete.

Remark 4.3. For the urn model with $r$ different colors, as mentioned in Remark 4.2, our method can also be used to prove that $X=\left(X_{1}, \ldots, X_{r}\right)$ is absolutely continuous. This requires a little modification and rewriting, i.e.

$$
\Omega_{\ell}=\left\{\omega: Y_{\ell i} \geq c m \text { for all } i=1, \ldots, r\right\}, \quad \Omega_{\ell, j}=\left\{\omega: Y_{\ell}=\left(Y_{\ell 1}, \ldots, Y_{\ell r}\right)=j\right\}
$$

and

$$
\sum_{t} \mathrm{P}\left(Y_{n+1}=j+c k \mid Y_{n}=j+c(k-t)\right) \leq 1-\frac{r-1}{n}+\frac{c^{\prime}}{n^{2}},
$$

where $j=\left(j_{1}, \ldots, j_{r}\right), k=\left(k_{1}, \ldots, k_{r}\right)$, and $t=\left(t_{1}, \ldots, t_{r}\right)$.

\section{Appendix A.}

In this appendix, more examples are illustrated, in Figure 3, to show the effects of different initial numbers of black balls $b$ and white balls $w$, and numbers, $m$, of balls drawn each time. As in Section 3, $\mathrm{M}(b, w, m)$ denotes our new model with initial data $(b, w, m)$ and beta $(\alpha, \beta)$ denotes a beta distribution with parameters $\alpha$ and $\beta$. 

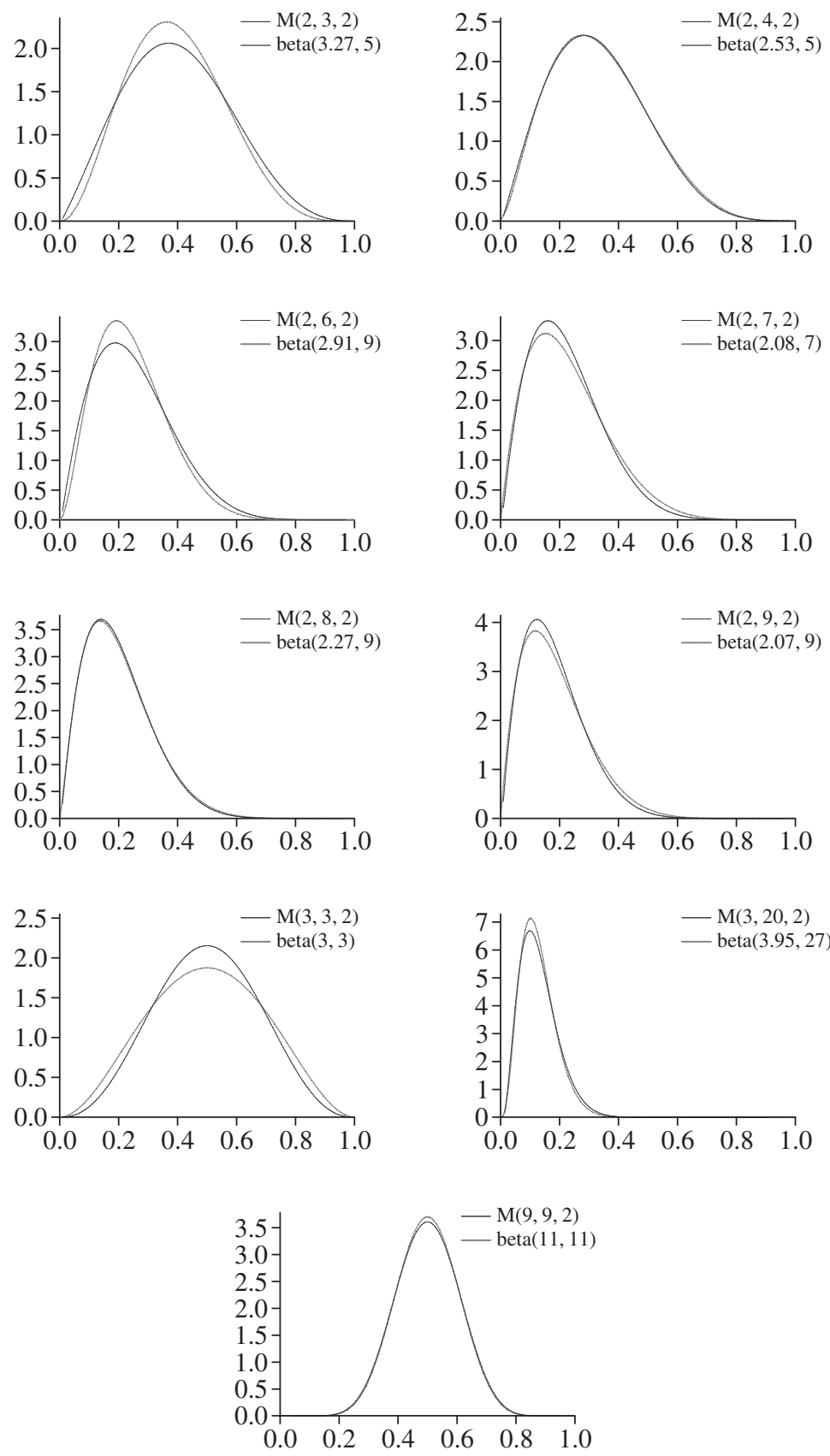

FIGURE 3: Nine examples with $n=100$.

\section{Acknowledgement}

The authors wish to thank the referee for helpful suggestions that improved the paper and made it more readable. 


\section{References}

[1] Bagchi, A. And PAL, A. K. (1985). Asymptoic normality in the generalized Pólya-Eggenberger urn model, with an application to computer data structures. SIAM J. Algebraic Discrete Meth. 6, 394-405.

[2] Billingsley, P. (1995). Probability and Measure, 3rd edn. John Wiley, New York.

[3] Eggenberger, F. And Pólya, G. (1923). Über die statistik verketteter vorgänge. Z. Angewandte Math. Mech. 1, 279-289.

[4] Feller, W. (1971). An Introduction to Probability Theory and Its Applications, Vol. II, 2nd edn. John Wiley, New York.

[5] Gouet, R. (1989). A martingale approach to strong convergence in a generalized Pólya-Eggenberger urn model. Statist. Prob. Lett. 8, 225-228.

[6] Gouet, R. (1993). Martingale functional central limit theorems for a generalized Pólya urn. Ann. Prob. 21, 1624-1639.

[7] Hall, P. ANd Heyde, C. C. (1980). Martingale Limit Theory and Its Application. Academic Press, New York.

[8] Hill, B., Lane, D. and Sudderth, W. (1980). A strong law for some generalized urn processes. Ann. Prob. 8, 214-226.

[9] Johnson, N. L. And Kotz, S. (1977). Urn Models and Their Application. John Wiley, New York.

[10] Kotz, S. and Balakrishnan, N. (1997). Advances in urn models during the past two decades. In Advances in Combinatorial Methods and Applications to Probability and Statistics, Birkhäuser, Boston, MA, pp. $203-257$.

[11] Maistrov, L. E. (1974). Probability Theory: a Historical Sketch. Academic Press, New York.

[12] Pemantle, R. (1990). A time-dependent version of Pólya's urn. J. Theoret. Prob. 3, 627-637.

[13] SchreIber, S. J. (2001). Urn models, replicator processes, and random genetic drift. SIAM J. Appl. Math. 61, 2148-2167.

[14] Stewart, I. (1989). Galois Theory, 2nd edn. Chapman and Hall, New York.

[15] WeI, C. Z. (1993). Martingale transforms with non-atomic limits and stochastic approximation. Prob. Theory Relat. Fields 95, 103-114.

[16] Wheeden, R. L. And Zygmund, A. (1977). Measure and Integral. Marcel Dekker, New York. 